\title{
Students' understanding and perceptions of the content of a lecture
}

\author{
Zdeslav Hrepic, Dean Zollman and Sanjay Rebello \\ Physics Department, Kansas State University, Manhattan, KS 66506 \\ zhrepic@phys.ksu.edu; dzollman@phys.ksu.edu; srebello@phys.ksu.edu
}

\begin{abstract}
In spite of advances in physics pedagogy, the lecture is by far the most widely used instructional format. We investigated students' understanding and perceptions of the content delivered during a physics lecture. Students participating in our study responded to a written conceptual survey on sound propagation. Next, they looked for answers to the survey questions in a videotaped lecture by a nationally known teacher. As they viewed the lecture, they indicated instances, if any, in which the survey questions were answered during the lecture. A group of experts (physics instructors) also participated in our study. We discuss students' and experts' responses to the survey questions.
\end{abstract}

\section{Introduction}

The lecture is perhaps the oldest instructional format that is commonly used today. Researchers' interest in issues related to classroom teaching has resulted in a variety of findings significant for improvements in instruction.(Cooper \& Simonds, 2003) However, educators are still concerned with how students learn in a traditional lecture.(Kvasz, 1997; Zollman, 1996) Although many novel instructional methods have been developed, it is unlikely that the lecture will soon be replaced as the most commonly used format. Therefore, the lecture deserves our attention.

\section{Motivation}

In our recent study on students' mental models of sound propagation,(Hrepic, Zollman, \& Rebello, 2002) we interviewed students using the same protocol before and after lecture-based instruction. An observation of the lecture by one of the authors (Dean Zollman) indicated that the instructor had explicitly answered some of the interview questions during the lecture. However, several interviewees stated that they were unable to find answers to the interview questions in the lecture, even though they specifically looked for them. This mismatch between the perceptions of students and experts regarding the content of the lecture motivated the research presented here.

\section{Goals}

Our research questions were:

- What kind of questions do students perceive as being answered in a lecture?

- How are students' perceptions related to their knowledge prior to the lecture?

- How do students' perceptions of the content of a lecture compare with those of experts?

\section{Methodology}

We interviewed 18 students in a conceptual physics class at Kansas State University. Over half of the students had taken high school physics. Students received extra credit worth $2 \%$ of the course grade for their participation. Sound propagation was the topic of the experimental lecture and the study was conducted soon after students had already completed their lectures and also taken an in-class exam on this topic.

In the experiment students viewed a videotaped lecture on the chosen topic, which was different from the one they had heard in class. We used a segment of a commercially available video lecture(Hewitt, 1991) on sound propagation by the author of the class text. (Hewitt, 1998) The duration of a lecture segment was less than a third of the normal class time. The fact that the lecture was given by a nationally known teacher was deemed to improve the possibility that students would find the lecture understandable. Both the lecturer and the students were native English speakers. Students had full control over video and 
there were not any typical classroom distractions such as noise etc. Therefore, the experimental situation had several important advantages compared to a typical classroom.

Before students viewed the video, they responded to a written survey on sound propagation. Survey questions ranged from those addressed explicitly in the video to those not addressed at all. The survey enabled us to gauge students' initial understanding of sound propagation. It also provided specific questions to which students were subsequently asked to find answers to in the video.

The following questions were on the survey:

Q1. Describe the nature/mechanism of sound propagation in air? [Answer: Sound is the propagation of the (longitudinal) vibration of medium particles. Or, sound is a pressure wave.]

Q2. Does the speed of the sound in air depend on temperature? [Answer: Yes. Sound propagates faster if the temperature is higher.]

Q3. Does the speed of propagation of sound depend on the motion of the source? [Answer: No. It depends only on medium properties.]

Q4. Does the speed of propagation of sound depend upon the medium? If so, how does the speed of sound generally compare between solids, liquids and gases. [Answer: Yes. Generally it is faster in solids than in liquids and faster in liquids than in gasses.]

Q5. Does sound propagate in a vacuum? [Answer: No. It needs a medium.]

Q6. Does sound affect a dust particle floating in front of the loudspeaker? If so, how? [Answer: Yes. It will vibrate longitudinally.]

While watching the video, students were asked to record the answer they perceived as being given in the video to each survey question. They were also asked to indicate, on a Likert scale, the extent to which the question was answered: 1 (Hint of the answer) to 5 (Answered completely).

After completely viewing the video, each participant was asked to record whether any further answers could be inferred from the video. This task aimed to determine questions whose answers the student perceived as being indirectly addressed, although not explicitly answered in the video.

Besides students, we also surveyed a group of experts using the same protocol. For this study experts were defined as M.S. or Ph.D. degree holders in physics. We also required the expert's mental model of sound propagation before the video lecture was undoubtedly the wave model. Two of 11 of the potential experts were disqualified as they did not satisfy this requirement. Nearly half of the experts were nonnative English speakers. Additionally, we asked the videotaped instructor, Paul Hewitt, to participate in the study.

\section{Data Analysis}

A uniform set of criteria were applied to analyze the data from all participants - students and experts. Due to the complexity of answers to Q1, we classified participants' answers in terms of their mental models of sound propagation determined from earlier research.(Hrepic et al., 2002)

\section{Results and Discussion}

As a reference point we started with responses from Dr. Hewitt:

Q1: Answered (rated 4/5)

Q2: Answered completely (rated 5/5)

Q3: Not answered

Q4: Answered

(rated 4/5)

Q5: Not answered

Q6: Partially answered (rated 2/5)

Results obtained from students and experts are shown in Table 1. Both students and experts perceived Q1, Q2 and Q4 as answered in the lecture. However, unlike students, experts in general perceived Q5 and Q6 as also answered in the lecture. Conversely, Q3 was perceived as addressed by five students though not by a single expert. In general, experts perceived questions as being answered more 
frequently than students did (except Q3 that no expert saw as answered). Similarly, for all but one question (Q2) experts rated the answers as being more complete than the students did.

In almost all cases the number of correct answers after the lecture is practically the same as the number of correct answers before the lecture. The exceptions are students' answers to Q2 and Q4. These questions were explicitly addressed in the lecture. Although the whole lecture segment was related to sound propagation, three students perceived that Q1 was not addressed at all. One student recorded the answer to Q1, artificially, so he did not address the nature of sound propagation. Only three students "upgraded" their models: Two of them from an incorrect to a less incorrect model and only one from an incorrect model to the correct model. For three students we could not determine with certainty the mental model that they used, but their responses were clearly inconsistent with the wave model. The remaining students retained their initial (incorrect) model after the lecture.

\begin{tabular}{|c|c|c|c|c|c|c|c|c|c|c|}
\hline \multirow[b]{2}{*}{ Qs } & \multirow[b]{2}{*}{$\begin{array}{c}\text { Viewers } \\
\text { group }\end{array}$} & \multicolumn{3}{|c|}{$\begin{array}{l}\text { Frequency at which the } \\
\text { participants saw the questions } \\
\text { addressed in the video lecture }\end{array}$} & \multicolumn{3}{|c|}{$\begin{array}{l}\text { Completeness with which } \\
\text { questions were addressed as } \\
\text { rated by participants }\end{array}$} & \multicolumn{3}{|c|}{$\begin{array}{l}\text { Correctness of the answers given by } \\
\text { participants during/after the video } \\
\text { lecture }\end{array}$} \\
\hline & & $\begin{array}{l}\text { Question } \\
\text { seen as } \\
\text { addressed } \\
\text { by }(\%)\end{array}$ & $\begin{array}{c}\text { Average } \\
\text { number } \\
\text { of times } \\
\text { addresse } \\
\text { d }\end{array}$ & $\begin{array}{l}\text { Mode } \\
\text { of } \\
\text { times } \\
\text { addre } \\
\text { ssed }\end{array}$ & $\begin{array}{c}\text { Comple } \\
\text { te-ness } \\
\text { rated by }\end{array}$ & $\begin{array}{c}\text { Avg } \\
\text { Comple } \\
\text { te-ness }\end{array}$ & $\begin{array}{c}\text { Mode of } \\
\text { complet } \\
\text { eness }\end{array}$ & $\begin{array}{l}\text { Answered } \\
\text { correctly } \\
\text { and with } \\
\text { relevance }\end{array}$ & $\begin{array}{l}\text { Answered } \\
\text { correctly } \\
\text { also before } \\
\text { the video }\end{array}$ & $\begin{array}{c}\text { Correct } \\
\text { answer } \\
\text { given } \\
\text { only as } \\
\text { an } \\
\text { inference }\end{array}$ \\
\hline \multirow{2}{*}{1} & $\begin{array}{c}\text { Students } \\
(\mathrm{N}=18)\end{array}$ & 15 (83\%) & 1.2 & 1 & 13 & 3.8 & 5 & 2 & 1 & $\mathbf{0}$ \\
\hline & $\begin{array}{c}\text { Experts } \\
(\mathrm{N}=9)\end{array}$ & $9(100 \%)$ & 2.1 & 1 & 8 & 4.5 & 5 & 9 & 9 & $\mathbf{0}$ \\
\hline \multirow{2}{*}{2} & $\begin{array}{c}\text { Students } \\
(\mathrm{N}=18)\end{array}$ & $18(100 \%)$ & 1.3 & 1 & 17 & 4.8 & 5 & 17 & 8 & $\mathbf{0}$ \\
\hline & $\begin{array}{c}\text { Experts } \\
(\mathrm{N}=9)\end{array}$ & $9(100 \%)$ & 2.4 & 2 & 8 & 4.7 & 5 & 9 & 8 & 0 \\
\hline \multirow{2}{*}{3} & $\begin{array}{c}\text { Students } \\
(\mathrm{N}=18)\end{array}$ & $5(27.8 \%)$ & 1 & 1 & 3 & 2 & N/A & 1 & 1 & 1 \\
\hline & $\begin{array}{c}\text { Experts } \\
(\mathrm{N}=9)\end{array}$ & $0(0 \%)$ & $\mathbf{0}$ & $\mathbf{0}$ & 0 & 0 & 0 & $\mathbf{0}$ & $\mathbf{0}$ & $\mathbf{0}$ \\
\hline \multirow{2}{*}{4} & $\begin{array}{c}\text { Students } \\
(\mathrm{N}=18)\end{array}$ & $18(100 \%)$ & 1.4 & 1 & 16 & 4.1 & 5 & 17 & 12 & $\mathbf{0}$ \\
\hline & $\begin{array}{c}\text { Experts } \\
(\mathrm{N}=9)\end{array}$ & $9(100 \%)$ & 3 & 3 & 8 & 4.8 & 5 & 9 & 8 & 0 \\
\hline \multirow{2}{*}{5} & $\begin{array}{c}\text { Students } \\
(\mathrm{N}=18)\end{array}$ & $3(16.7 \%)$ & 1 & 1 & 2 & 2.3 & N/A & 2 & 2 & 2 \\
\hline & $\begin{array}{c}\text { Experts } \\
(\mathrm{N}=9)\end{array}$ & $7(77.8 \%)$ & 1 & 1 & 1 & 3 & N/A & 7 & 7 & 7 \\
\hline \multirow{2}{*}{6} & $\begin{array}{c}\text { Students } \\
(\mathrm{N}=18)\end{array}$ & $3(16.7 \%)$ & 1 & 1 & 4 & 2 & 2 & 2 & 1 & 1 \\
\hline & $\begin{array}{c}\text { Experts } \\
(\mathrm{N}=9)\end{array}$ & $7(77.8 \%)$ & 1 & 1 & 5 & 2.2 & 1 & 6 & 6 & 3 \\
\hline
\end{tabular}

Table 1: Results from students and experts 
There were a total of 20 instances (11 students and 9 experts) in which the participants decided after completely viewing the video, that an answer to a question could be inferred from the video. In all of these cases correct inferences were made only by participants (students and experts) who answered the question correctly before viewing the video.

In comparing the ways in which experts and students see questions as being answered on the video, only the instructor (Dr. Hewitt) and the most experienced expert perceived the questions as being addressed similar to the way in which students did. This result appears to indicate that more experienced teachers have a better sense of the ways in which students might perceive the content of a lecture.

When the nature of their answers is examined, the following traits are observed. Students may...

1. concentrate on details in the instructor's statements. (e.g. "Sound travels faster through the steel than through the lead.")

2. record details incorrectly. (e.g. "Sound travels four times faster in steel and about two times faster in water [than in air].”)

3. hear/understand exactly the opposite of what the instructor said. (e.g. "Sound propagates faster in cold air. Slower in a warm air.”)

4. hear what was not said. (e.g. "The sound molecules vibrate back and forth.”)

5. make inappropriate generalizations. (e.g. "In a liquid... sound would move four times faster than when it is not in a liquid")

6. create false positive answers. (e.g. "Sound bounces back and forth ...so the dust particle will move back and forth."

7. perceive the incorrect answer when no answer is given to the question. (e.g. "If the source is moving fast ... you'll hear it faster.")

8. correctly repeat instructors' statements but do not make sense of them. (e.g. "He [the instructor] was just talking about the way the sound moves. When molecules start moving, they're vibrating back and forth and they hit the next one and the next one ... [Sound is] just traveling with those, I guess. I don't know. It's just traveling with that. Like being carried with each vibrating molecule. ... I'm just in the dark with this whole sound thing.)

9. correctly repeat the instructor's statement without realizing that it does not make sense to them. (e.g. "Molecules hit one another until they reach the person." Interviewer: How is sound related to these molecules hitting each other? ... Student: "What do you mean? ...I don't know. I mean I don't think every molecule just kind of transfers...I don't know. I didn’t think about it.”)

10. correctly repeat the instructor's statements but interpret them differently than intended. (e.g. The students understood statements about vibration of molecules when given an example of a room full of vibrating ping pong balls so that sound is an autonomous entity which is different from the medium which moves by using vibration of medium molecules.)

11. ...hear "what makes sense" and overlook what was actually stated. (e.g. "[The dust] particle vibrates up and down," (the same answer as given before the interview). Follow-up by Interviewer: "So what did he [instructor] say about the direction of vibration? Do you remember?” Student: (Pause) What do you mean?” Interviewer: How did you conclude that they will vibrate up and down?” Student: 12. (Pause) Just...it wouldn’t...it wouldn’t make sense to vibrate...They couldn’t vibrate sideways.”)

We now discuss the effect of earlier answers on student understanding of the lecture content. Examples are omitted from this section due to lack of the space. With respect to their earlier answers students may...

1. stick to their previous background ideas although they change the specific answer.

2. $\quad$ keep their initial (incorrect) model in identical form.

3. inappropriately incorporate new information into the existing (incorrect) model.

4. use the same terminology as experts do, with a very different meaning attached to it before and after the lecture.

5. be confused more after than before the lecture. 


\section{Conclusions}

In general we found in our study that...

- Students correctly notice answers that are simple and explicitly stated, preferably multiple times. Otherwise, they may try to make sense of things in a ways not intended by the instructor.

- Students make incorrect inferences. Correct inferences were made in this study only when the student (or experts) knew it previously.

- Experts tend to believe that more was delivered than actually was and frequently perceive questions more addressed than the students do.

All of the above findings are important for a lecturer to bear in mind because the aforementioned problems with students' understanding were observed in a situation that had significant advantages to a classroom lecture.

\section{Acknowledgements}

This work is supported in part by NSF grant \# REC-0087788.

The authors wish to thank Dr. Paul Hewitt for his kind participation in this research. His input was invaluable for analysis of our data.

\section{References}

Cooper, P. J., \& Simonds, C. J. (2003). Communication for the clasroom teacher. Boston, MA: Allyn and Bacon.

Hewitt, P. G. (1991). Vibrations and Sound II [Video tape]: Addison-Wesley.

Hewitt, P. G. (1998). Conceptual Physics (8th ed.). Reading, MA: Addison-Wesley.

Hrepic, Z., Zollman, D., \& Rebello, S. (2002). Identifying students' models of sound propagation. Paper presented at the 2002 Physics Education Research Conference, Boise ID.

Kvasz, L. (1997). Why don't they understand us? Science and Education, 6, 263-272.

Zollman, D. (1996). Millikan Lecture 1995: Do They Just Sit There? Reflections on Helping Students Learn Physics. American Journal of Physics, 64, 114-119. 